\title{
Global Skin-Friction Measurements Using Particle Image Surface Flow Visualization and a Luminescent Oil-Film
}

\author{
Nicholas Husen ${ }^{1}$ \\ School of Aeronautics and Astronautics \\ Purdue University, West Lafayette, IN 47906 \\ Nettie Roozeboom ${ }^{2}$ \\ NASA Ames Research Center, Moffett Field, CA 94035 \\ Tianshu Liu ${ }^{3}$ \\ Western Michigan University, Kalamazoo, MI 49008 \\ and \\ John P. Sullivan ${ }^{4}$ \\ School of Aeronautics and Astronautics \\ Purdue University, West Lafayette, IN 47906
}

\begin{abstract}
A quantitative global skin-friction measurement technique is proposed. An oil-film is doped with a luminescent molecule and thereby made to fluoresce in order to resolve oil-film thickness. Particle Image Surface Flow Visualization (PISFV) is used to resolve the velocity field of the surface of the oil-film. Skin-friction is then calculated at location $\vec{x}$ as $\tau(\vec{x})=\mu \vec{v}_{h}(\vec{x}, t) / h(\vec{x}, t)$, where $\vec{v}_{h}(x, t)$ is the velocity field of the surface of the oilfilm, $h$ is the thickness of the oil-film, $t$ is time, and $\mu$ is the dynamic viscosity of the oil. The data collection and data analysis procedures are explained, and preliminary experimental skin-friction results for flow over the wing of NASA's Common Research Model are presented.
\end{abstract}

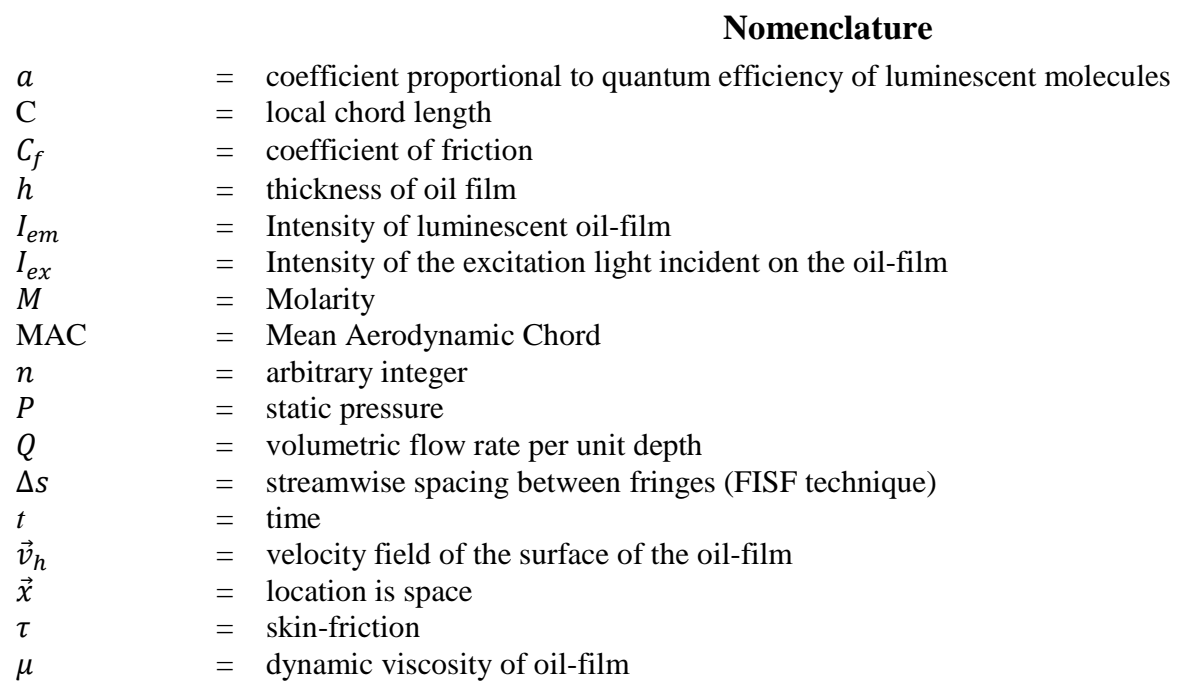

\footnotetext{
${ }^{1}$ Graduate Student, School of Aeronautics and Astronautics, 701 West Stadium Avenue. Student Member AIAA.

${ }^{2}$ Aerospace Engineer, Experimental Aero-Physics Branch, NASA Ames Research Center, Moffett Field, CA 94043.

${ }^{3}$ Professor, Department of Mechanical and Aeronautical Engineering, G-220, Parkview Campus, MI 49008, Senior Member AIAA.

${ }^{4}$ Professor, School of Aeronautics and Astronautics, 701 West StadiumAvenue. Fellow AIAA.
} 


\section{Introduction}

$S^{k}$ kin-friction, or surface shear-stress, constitutes as much as fifty percent of the drag on commercial transport aircraft at cruise and can cause loadings on structures such as wing surfaces, flaps and landing gear. Also, the CFD community relies heavily on skin-friction values when setting important parameters such as $u_{\tau}$ and $y^{+}$-parameters which when perturbed only slightly can give rise to very different flow field predictions. However, skin friction remains a difficult quantity to measure experimentally. Many point-based skin-friction measurement techniques have been fairly well matured, including oil-film interferometry (Naughton and Brown (1996), Naughton and Hind (2013)), MEMS (Etebari (2008), Kahng et al. (2002)), and liquid crystals (Reda et al. (1998)). Relevant to the present article is work done by Tianshu Liu and his colleagues to develop a global luminescent oil-film skin-friction meter which they used to experimentally determine skin friction lines for several juncture flows, delta wings, and NACA airfoils (Liu et al. (1998, 2008)), their work on optical flow algorithms (2008), and the work by Mosharov and his colleagues developing Particle Image Surface Flow Visualization (PISFV) techniques (Mosharov et al. (2006), Mosharov and Radchenko (2012)). The current article aims to build upon the existing literature to develop a skin-friction meter which can produce quality quantitative skin friction data with unprecedented resolution-as many as several hundred experimentally determined data points per square inch.

\section{SUMMARY OF TECHNIQUE}

The technique detailed herein is summarized as follows. A thin luminescent oil-film is applied to the surface of a model. The thickness of the oil-film will later be detected by measuring the intensity of the emissions from the luminescent molecules in the oil-a brighter region corresponds to thicker oil. Fluorescent particles are applied to the surface of the oilfilm. So that the emissions from the luminescent particles and the emissions from the fluorescent oil-film are separable via optical bandpass filters, the particles emit at a wavelength that is different from that emitted by the fluorescent oil-film. The tunnel is operated and images of the oil-film/particle-field are taken on regular intervals-approximately six frames per minute-alternating between an optical filter for viewing emissions from the particles and an optical filter for viewing emissions from the oil-film.

It is emphasized: Two sets of data (images) are taken during this experiment. One set of images reveals the particle locations on the surface of the oil-film. The second set of images captures the luminescent intensity of the fluorescent emissions from the oil-film.

The velocity field of the surface of the oil-film is revealed by applying optical flow methodologies to pairs of particle images. The luminescent intensity of the emissions from the oil-film is directly proportional to the oil-film thickness. Therefore, measuring the luminescent intensity of the oil-film reveals a global relative thickness distribution of the oil-film. Finally, letting $\mu$ denote the dynamic viscosity of the oil, $h$ denote the thickness of the oil-film, $t$ denote time, and $v_{h}$ denote the velocity field of the surface of the oil-film, the skin-friction magnitude is calculated at location $\vec{x}$ and as

$$
\vec{\tau}(\vec{x})=\frac{\mu \vec{v}_{h}(\vec{x}, t)}{h(\vec{x}, t)} .
$$

\section{THEORY}

It is shown in Massey (1970) that the volumetric flow rate of a thin oil-film of height $h$ subjected only to a pressure gradient $d P / d x$ and skin-friction $\tau$ is given by

$$
Q=\frac{\tau h^{2}}{2 \mu}-\frac{h^{3}}{3 \mu} \frac{d P}{d x} .
$$

In the absence of a pressure gradient or for a sufficiently thin oil-film $(h \ll 1)$ the second term drops out and

$$
Q=\frac{\tau h^{2}}{2 \mu} .
$$

Using a control volume approach, Tanner and Blows (1976) show that the velocity $v_{h}$ of a particle at the surface of the oilfilm is then given by

$$
v_{h}=\frac{\partial Q}{\partial h}=\frac{\tau h}{\mu} .
$$

Rearranging, we observe that skin-friction may be expressed as

$$
\tau=\frac{\mu v_{h}}{h} .
$$

Therefore, in order to determine skin-friction for situations in which the pressure gradient is negligible or the oil-film is sufficiently thin one needs to know the thickness of the oilfilm, $h$, the velocity of the surface of the oil-film, $v_{h}$, and the dynamic viscosity of the oil-film, $\mu$.

The oil-film is doped with a luminescent molecule so that it fluoresces under $450 \mathrm{~nm}$ light. The luminescent intensity of the fluorescence is directly proportional to the film's thickness, $h$. Letting $I_{e x}(\vec{x})$ denote the intensity of the excitation energy being cast upon the oil-film at location $\vec{x}$, $I_{\text {em }}(\vec{x})$ denote the intensity of the light emitted by the fluorescent oil-film at location $\vec{x}$, and by letting $a$ be a constant capturing the quantum efficiency of the dye molecule and the concentration of the dye in the oil,

$$
I_{e m}(\vec{x}, t)=a I_{e x}(\vec{x}) h(\vec{x}, t)
$$

or

$$
h(\vec{x}, t)=\frac{I_{e m}(\vec{x}, t)}{a I_{e x}(\vec{x})}
$$

The velocity of the surface of the oil-film, $v_{h}$, is found by distributing particles on the surface of the oil-film and imaging their motion under testing conditions (wind-on). The captured frames are then processed using optical flow 
algorithms to produce a velocity field for the surface of the oil-film. Finally, the dynamic viscosity of the oil is assumed to be known, although a temperature-viscosity calibration and model-mounted thermocouples would increase accuracy.

\section{Experimental Setup}

The measurement system consists of an excitation source, a luminescent oil-film, the fluorescent particles, two optical filters, and one camera. A schematic representation of the system is shown in Figure 1.

The viscosity of the silicone oil is chosen to be 10,000cSt. This highly viscous oil is chosen because it evolves very slowly, allowing the time between successive images to be as large as 10 to 20 seconds. This large time period provides time for the optical filters to alternate between a $550 \mathrm{~nm}$ bandpass filter capturing the particle locations and a $650 \mathrm{~nm}$ bandpass filter capturing the luminescent intensity of the oil-film. The highly viscous oil also has the favorable characteristic that it remains in the region of interest through wind-tunnel start-up and through several different experimental conditions. It must be applied as a very thin layer $(\sim 100 \mu \mathrm{m})$ in order to avoid wind-driven ripples at the oil-air interface. This thin film of oil also puts the oil-film within the range of thicknesses for which the oil-film thickness-luminescent intensity relationship is linear.

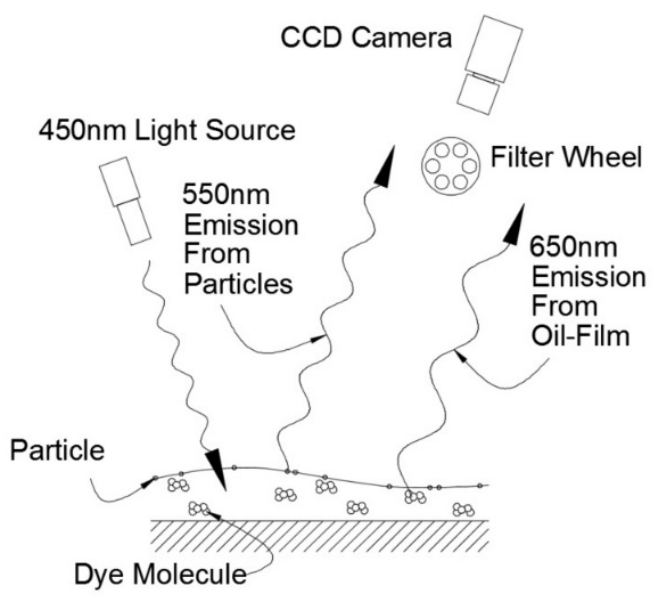

Figure 1. Major components of the experiment system.

It is particularly important that the experimenter is able to discern between emissions from the particles and emissions from the luminescent oil-film. The two substances must therefore emit at starkly different wavelengths. Further, there is a lower bound on the wavelength of the desired excitation source because the experimental community would like to be able to pass through windows typical of wind tunnel test section the emissions from the excitation source. The excitation source was therefore chosen to emit over a bandwidth centered at $450 \mathrm{~nm}$. The particles were chosen to emit at a bandwidth centered around $550 \mathrm{~nm}$, and the dyemolecule for the oil-film was chosen to emit over a bandwidth centered around 650nm. The particles are a PF-33 Palm-Leaf Green pigment from Risk Reactor Inc., and the dye for the silicone oil is Pyrromethene 650 from Exciton.
Pyrromethenes are excellent candidates for a dye in silicone oil as they are soluble in silicone oil and in toluene, which is also a solvent for silicone oil. Optical bandpass interference filters are used to separate the emissions from the particles from the emissions from the luminescent oil-film (and vice versa). These filters are from Melles Griot, are centered at $550 \mathrm{~nm}$ and $650 \mathrm{~nm}$ and have full-width half-max bandwidths of 40nm each. The camera is a Photometrics CoolSnap K4 4MP camera, chosen largely due to its availability. The excitation source is four 1650mW LED lamps from LED Supply. The LEDs emit over a bandwidth that is fairly narrow and centered at $450 \mathrm{~nm}$. The camera and lights were mounted in the plenum above the test section.

\section{EXPERIMENTAL PROCEEDURE}

This section is designed to outline the procedure one must walk through in order to collect skin-friction data using the method detailed herein.

\section{PREPARATION OF OIL, DYE, AND PARTICLES}

Pyrromethene laser dye is added to $10,000 \mathrm{cSt}$ silicone oil to create a luminescent oil that has a dye molarity of about $10^{-3} \mathrm{M}$. This is achieved as follows:

An empty $50 \mathrm{~mL}$ jar is placed on a balance and its mass is recorded. While on the balance, about three $\mathrm{mL}$ of $10,000 \mathrm{cSt}$ silicone oil is added to the jar. The new mass is recorded. The oil must be doped with the Pyrromethene molecules, and while Pyrromethene is soluble in silicone oil, it is much more readily dissolved into toluene. Approximately 1/1000g of Pyrromethene is weighed out and dissolved into toluene in a $25 \mathrm{~mL}$ volumetric flask. Some of this solution is pipetted into a $5 \mathrm{~mL}$ volumetric flask, and these five milliliters of solution are rinsed with toluene into the jar containing approximately three $\mathrm{mL}$ of silicone oil. Toluene is then added in order to reduce the viscosity so that an airbrush is able to dispense the solution. Importantly, after the oil-film is applied, the toluene will evaporate and only the dye and oil will remain on the model.

The particles are applied by stirring them into toluene and spraying them through a second airbrush onto the surface of the model. A solution of toluene and particles is prepared: Approximately $0.001 \mathrm{~g}$ particles are added to about $100 \mathrm{~mL}$ of toluene. This ratio is not particularly important as long as the researcher monitors the application and distributes the desired number of particles per unit area. The desired number of particles per unit area depends on the spatial resolution required in measurements. Suitably high particle density is preferred for optical flow computation to extract highresolution velocity fields (Liu and Shen (2008)).

\section{FIDUCIALS}

Fiducials are placed on the model in known locations in order to enable camera calibration. Also, because the PISFV-LOF method measures sub-pixel displacements of particles, image pairs need to align perfectly. Several fiducials are used to register image pairs with each other so that only the motion of the particles relative to the model is detected.

\section{REFERENCE IMAGES}

During the experiment several images are taken of the luminescent oil-film. These images are used to estimate 
$I_{e m}(\vec{x}, t)$. In order to prevent unwanted signal from being captured by the camera a $650 \mathrm{~nm}$ optical bandpass interference filter is used for this data collection. However, some photons that are not emissions from the luminescent oil-film make it through. One must take care, then, to subtract the signal that is detected by the camera in the absence of the luminescent oil-film. Immediately before the luminescent oil-film is applied, a sequence of images is captured and averaged to provide an image of the background signal that is received by the camera. This image will be subtracted from subsequent images of the luminescent oil-film taken during the experiment.

The intensity of the excitation light can vary spatially in the region of interest. This directly impacts the fluorescent emission from the luminescent oil-film. Before the experiment, a diffuse white surface such as a sheet of paper is placed over the region of interest. This surface must lie flat against the testing surface. With the lights arranged just as they would be during the wind-tunnel run, a sequence of images is captured and averaged. For this, the optical filters are removed, and the integration time and aperture are adjusted such that the camera is nowhere saturated. The resulting image provides a scalar, $I_{e x}(\vec{x})$, by which one may later scale the luminescent oil-film images to correct for spatially varying excitation energy.

If in order to create different testing conditions the test article must be physically moved during the experiment (such as to adjust the angle of attack), then the set of reference images (the oil-film background image and the excitation energy distribution image) must be generated for each position.

\section{APPLICATION OF OIL AND PARTICLES}

In order to avoid contamination, two separate airbrushes are employed-one for the luminescent oil-film, and one for the particles. The fairly low viscosity oil-Pyrromethene-toluene solution is transferred into the container used by the first airbrush, and a very light dusting of the solution is applied to the testing region. Sufficient time is then allowed to pass so that the toluene fully evaporates. The particle-toluene solution is then transferred into the container for the second airbrush and, making sure that the particles stay suspended in the toluene, the region of interest is dusted with the particles. The researcher should monitor the process through an optical filter that permits the green particles to be readily seen. Time is again allowed to pass so that the toluene evaporates.

\section{DATA COLLECTION}

The wind tunnel is operated. Once the conditions in the tunnel are on point, data collection may begin. Images are acquired at a rate not exceeding one frame per five seconds (0.2fps). The optical filter is alternated between $550 \mathrm{~nm}$ to $650 \mathrm{~nm}$ for each image, producing two distinct data sets. This is illustrated in Figure 2. Even numbered frames are images of particles and odd number frames are images of the luminescent oil-film (or vice versa). After at least 50 images have been acquired, the condition in the wind tunnel may be altered and another data set may be acquired.

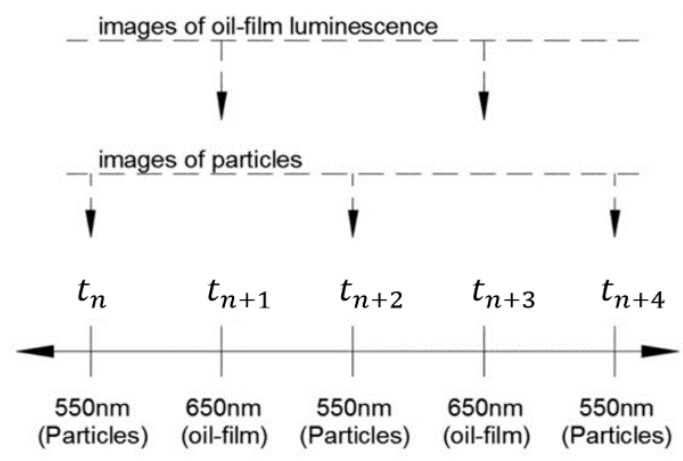

Figure 2. Illustration of image sequence. Optical filters alternate so that particle images and luminescent oil-film images are acquired in alternating frames.

\section{DATA PROCESSING}

Conducting the experiment generates four data files (sets of images):

1. Oil-film background images

2. Light distribution images

3. Particle evolution

4. Oil-film thickness evolution

The data sets are used as follows:

The oil-film background image is subtracted from each of the luminescent oil-film images, leaving behind an image $I_{e m}$ which is much more closely an image of only the emissions from the luminescent oil-film.

The light distribution image, denoted $I_{e x}$, is scaled such that the peak intensity value is around 1 . When one divides a luminescent oil-film image taken during the experiment (wind-on) by the light distribution image $I_{e x}$, the resulting image is an image which represents the luminescent intensity of a region excited uniformly by the excitation source. This is the relative thickness distribution $h=\frac{I_{e m}}{a I_{e x}}$. Since this is the thickness distribution up to a scalar, the constant $a$ is set to 1 $(a=1)$. A typical thickness distribution image $h$ is shown in Figure 3. 


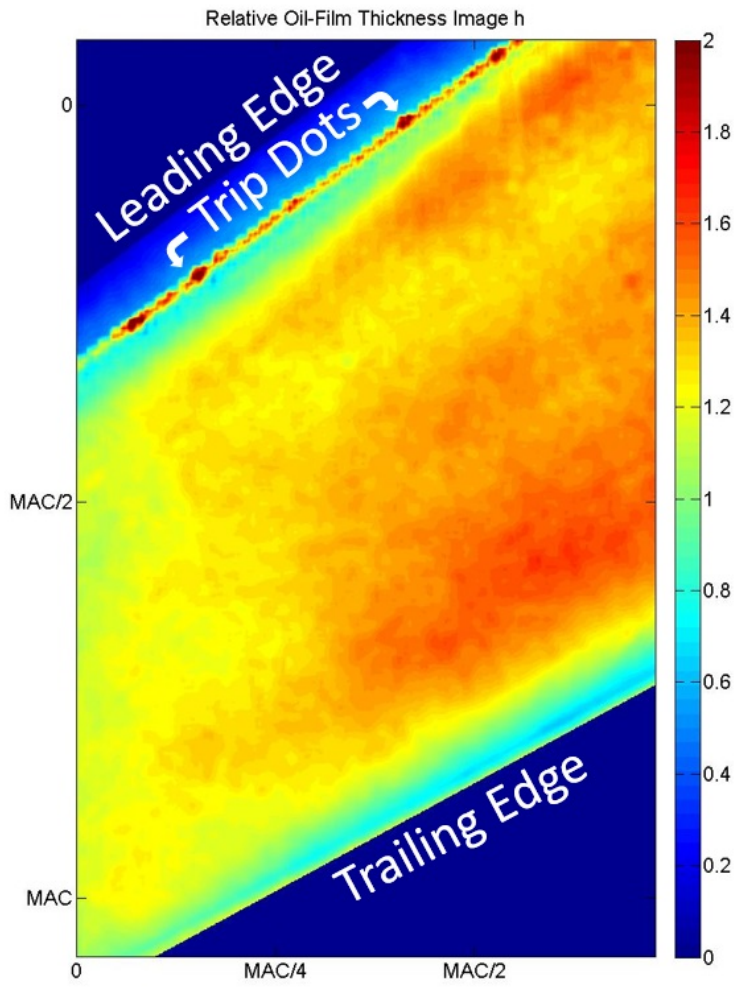

Figure 3. Relative oil-film thickness map. (e.g. An intensity value of 2 corresponds to a region of oil twice as thick as the region to which an intensity value of 1 would correspond.)

Because sub-pixel accuracy is important and because the relative positions of the model and the camera tend to change during the experiment due to tunnel vibrations, the particle images must be registered to each other. The centroids of the fiducials are identified and the images are translated into alignment. The particle images are then processed through a suite of optical flow codes (Liu and Shen, (2008)). The resulting velocity field, scaled appropriately, describes the motion of the surface of the oil-film in meters per second. A typical particle image is shown in Figure 4.

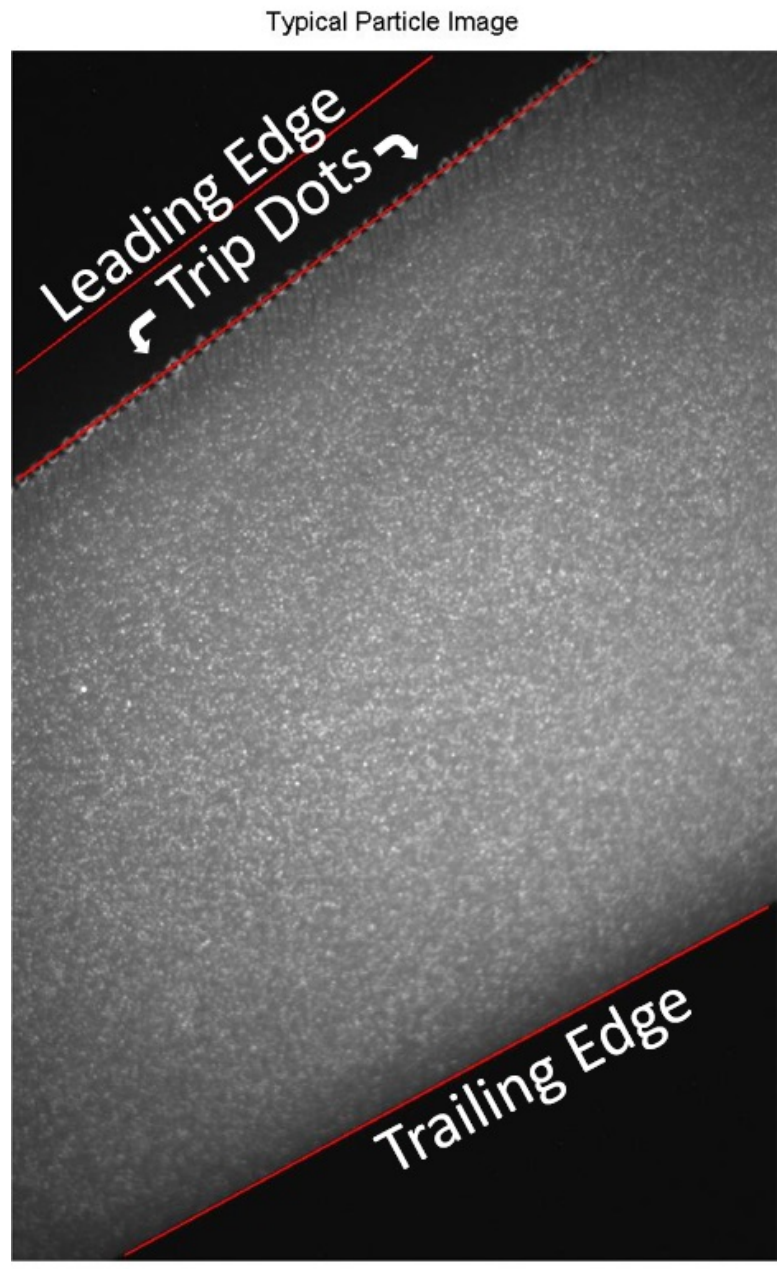

Figure 4. Typical Particle Image

An oil-film relative thickness image $h$ is taken from between the two images that were used for the optical flow process. That image is registered to the particle images and is used to complete the right hand side of Equation 1.

\section{Results}

Skin-friction measurements were made on a portion of the main wing of a scale model of NASA's Common Research Model (CRM) - a generic transport aircraft which was developed for the fourth AIAA Drag Prediction Workshop. The model used for the data in this paper is a 3\% scale model with its wings permanently deflected to match the shape of the wings at (full scale) cruise. The vehicle was mounted at 4 degrees angle of attack, and the tunnel was operated such that the Reynolds number based on the mean aerodynamic chord was about 675,000 .

The section of the wing used for this study is centered at $75 \%$ of the half-span from the aircraft's centerline and has a width equal to just over half of the main wing's mean aerodynamic chord. The location of this section is illustrated in Figure 5. 


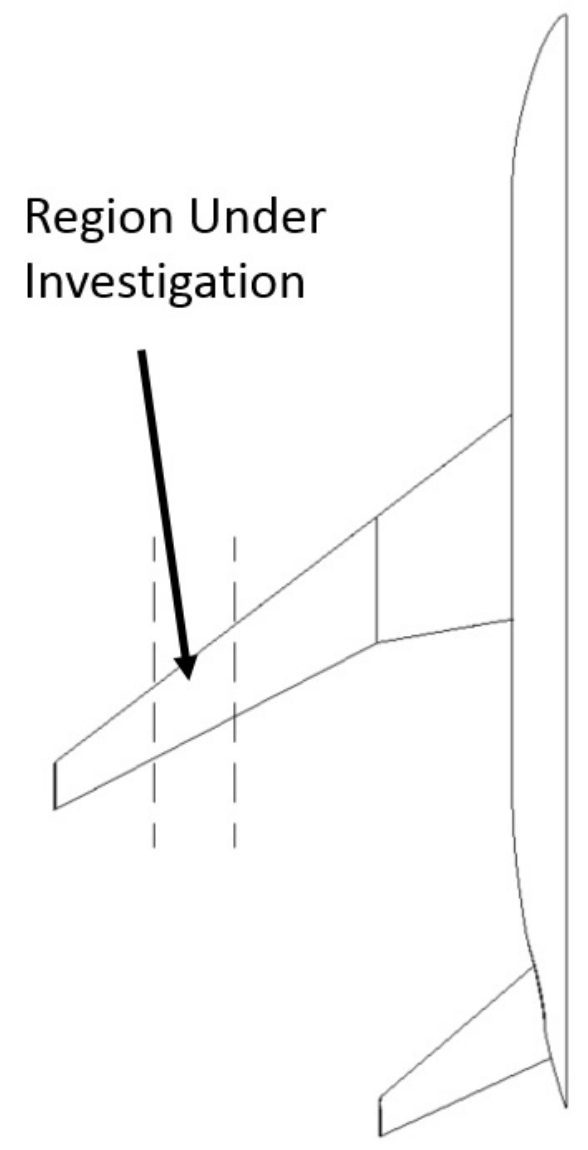

Figure 5. Schematic of NASA's Common Research Model (CRM) detailing where on the model data was taken.

The model had trip-dots installed about $0.1 c$ from the leading edge, where $c$ is the local chord length. The trip dots give way to significant streamwise vorticity that postpones or prevents the onset of separation (shown in preliminary flowvisualization data at NASA Ames). The streamwise vorticity is readily seen in a developed oil-film downstream of the trip dots. This is depicted in Figure 6. All data shown in this article is taken entirely downstream of the trip dots.
Close-Up of Oil-Film Downstream of Trip Dots

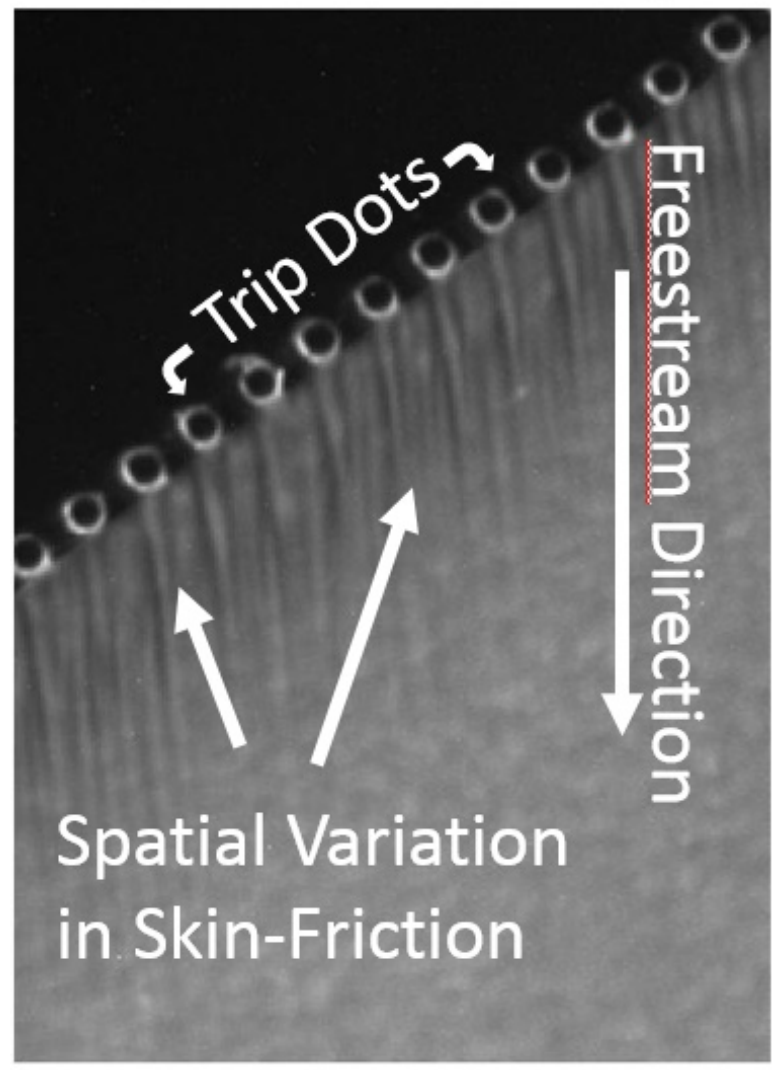

Figure 6. Developed oil-film downstream of trip dots. Skinfriction is seen to vary radically in the spanwise direction.

At the conditions described in this article, the flow was everywhere attached. Ahead of the trip-dots the spanwise component of the flow is significant, and aft of the trip-dots the flow is largely streamwise, turning outboard only in the aft-most five percent of the chord. A flow visualization was produced by allowing the flow to shear an oil-film upon which conglomerations of fluorescent pigment were initially at rest. The resulting streaklines are visible in Figure 7a. Figure $7 \mathrm{~b}$ is a schematic of the model and illustrates the approximate placement of the camera for the capture of the image in Figure 7a. Skin-friction lines produced using optical flow techniques are shown in Figure 8.

Skin-friction data was collected using the Particle Image Surface Flow Visualization-Luminescent Oil-Film (PISFV$\mathrm{LOF}$ ) technique proposed in this paper and by using the Fringe-Imaging Skin-Friction (FISF) technique. 


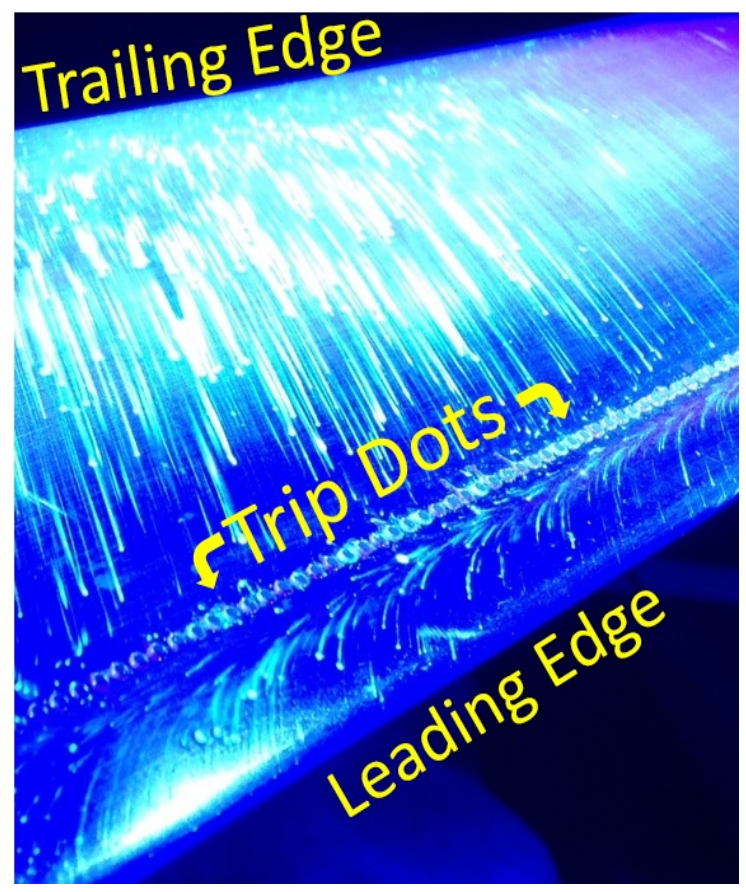

Figure 7a. An image of surface streaklines formed through the shearing of fluorescent pigment conglomerations on the surface of an oil film. There exists a significant spanwise component to the flow just upstream of the tripdots and also along the trailing edge.

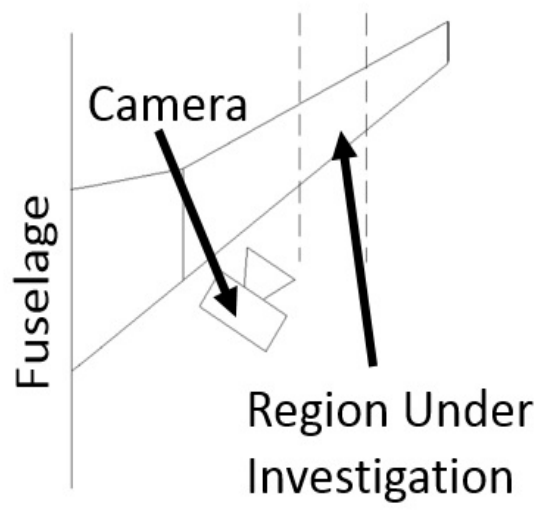

Figure $7 b$. Diagram showing the perspective from which the image in Figure 4. was taken.

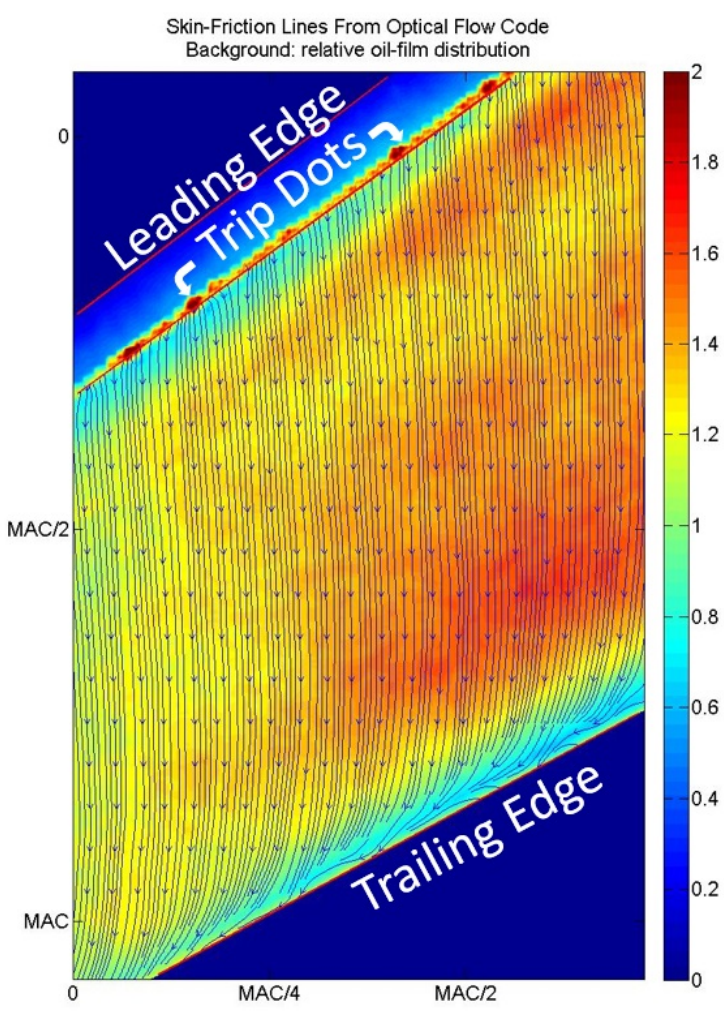

Figure 8. Skin-friction lines produced from a pair of particle images using optical flow techniques. An oil-film thickness distribution image, $h$, is in the background.

The FISF technique is a well-established, minimally intrusive, measurement technique and was used to measure skin friction on a small section of the wing. The skin friction is found through application of a simple expression based on lubrication theory relating fringe spacing to skin friction. Small drops of oil, of known viscosity, are placed on the wind tunnel model surface. The wind tunnel is turned on causing the oil to spread, forming a very thin wedge, of nearly linear profile. After the flow is turned off, an extended quasimonochromatic light source is used to illuminate the surface. Light reflecting from the top surface of the oil will interfere with light reflected from the surface of the wind tunnel model creating an interference pattern. This pattern is then imaged using a digital camera. The distance, $\Delta S$, between the fringes is proportional to the thickness of the oil, which is proportional to the skin friction. The skin friction coefficient magnitude is calculated by using fringe spacing found from a regression algorithm followed by a conversion of the pixelbased fringe spacing to physical coordinates using photogrammetry. The PC-based application, CXWIN5G, developed by Gregory G, Zilliac was used in this study. More information about this technique is discussed in the Zilliac (1996), and past application in Zilliac (2011) and Ross (2013).

Each drop of oil generates one data point. For this experiment, only 24 skin friction values were collected during one run using the FISF technique. Ideally, more FISF data points would be collected for comparison between the two techniques discussed. 
Figure 9a shows a raw image of the sheared droplets of oil. The fringes from the destructive interference are evident. Figure $9 \mathrm{~b}$ is a close-up view of the boxed region from Figure 9a. The greater values of skin-friction near the trip dots give way to a thinner wedge of oil and therefore a fringe pattern that is more spaced out. The smaller values of skin-friction further downstream give rise to more closely spaced fringes.

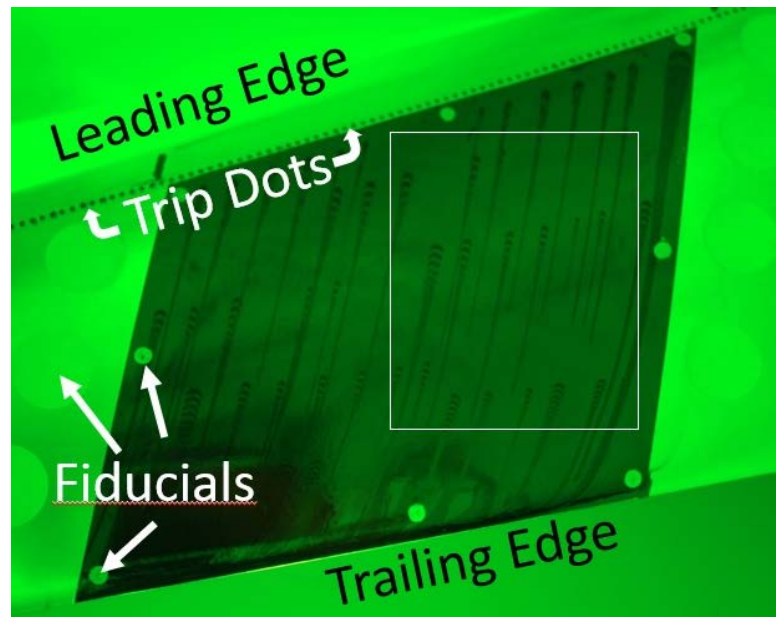

Figure 9a. Raw data image taken for fringe-imaging skinfriction (FISF) technique. The fringes in the sheared drops of oil are visible. The white box is the region cropped for further study in Figure $9 b$.

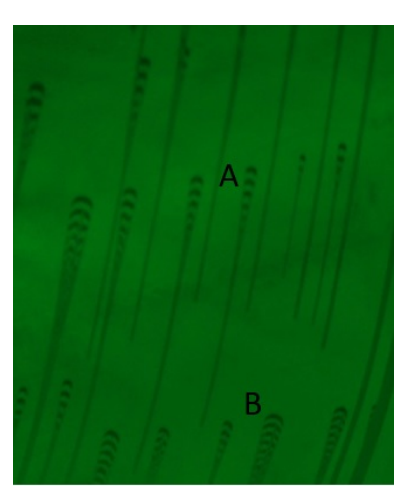

Figure $9 b$. The distance, $\Delta s$, between the destructive interference bands is proportional to the thickness of the oil, which is proportional to the skin-friction. This is evident in the image to the left, where the destructive interference bands are far apart in the region of high skinfriction (A), but closer together in the region of lower skin-friction (B).

The data generated by the PISFV-LOF technique is a relative skin-friction field. This data is therefore scaled to come as close as possible (in a least-squares sense) to FISF-measured skin-friction values. Figure 10 depicts the $C_{f}$-values determined by the PISFV-LOF technique and the locations of the 24 points at which FISF data was taken.

After being scaled to a best fit of the FISF data, the PISFVLOF data shows encouraging closeness to the skin-friction values determined at the 24 discrete FISF locations. The percent by which the PISFV-LOF data is different from the FISF data is shown in Figure 10. The discrepancy is as high as $26 \%$ at one location near mid-chord, but as low as $1.6 \%$ at another mid-chord location. In general the discrepancy seems to be around $2 \%$ to $15 \%$.

There exists uncertainty in each technique. The viscosity of the oil varies by about $1 \%$ for each degree Fahrenheit change in temperature. This translates directly through Equation 1 to a $1 \%$ change in skin-friction values per degree Fahrenheit. As this particular tunnel draws ambient air from the room, models can change temperature by as much as 20 degrees Fahrenheit during a single run, and since for each set of measurements (FISF and PISFV-LOF) temperature was not accounted for, the discrepancies shown in Figure 10 are within the expected range of values. Uncertainties also are introduced because of the large spatial gradients in skinfriction magnitude. Near the trip dots the skin-friction values decay steeply as one moves aft. A slight imperfection in the measurement of a data-point location therefore has a fairly significant effect on the accuracy of the associated skinfriction value.

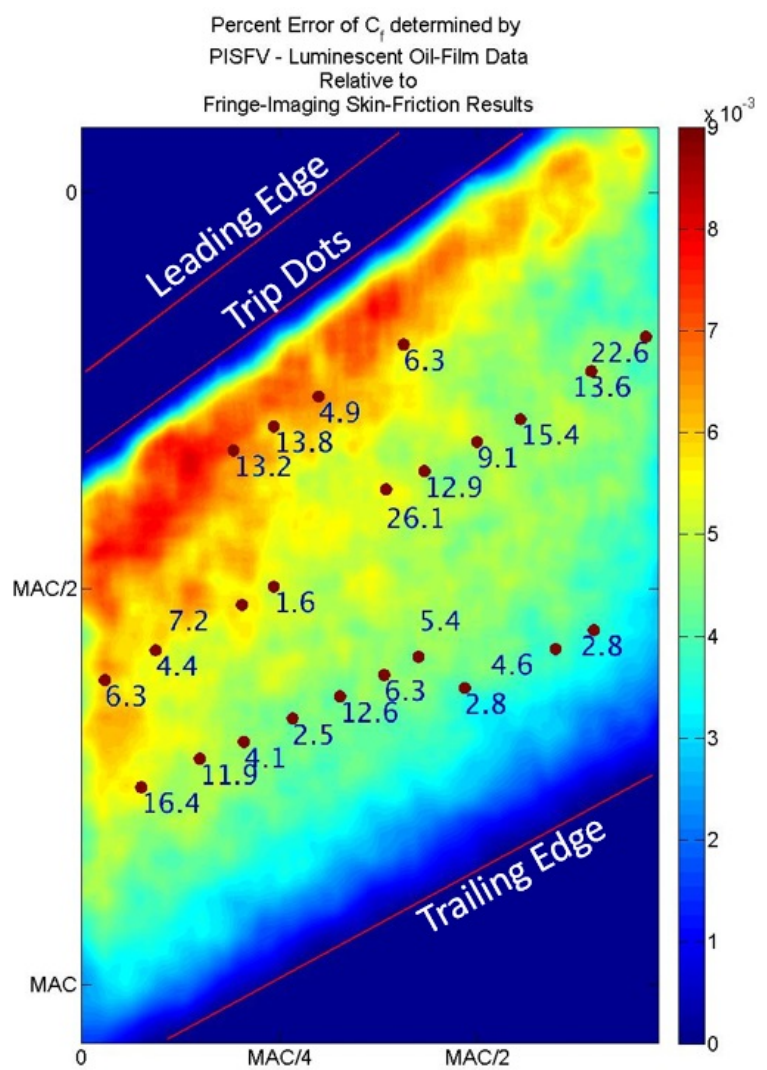

Figure 10. Map showing $C_{f}$-values from the PISFVLuminescent Oil-Film technique together with the percent by which those values differ from the $C_{f}$ values obtained using the FISF technique.

Streamwise sections showing the skin-friction profiles measured by each technique are presented in Figures 11a and 11b. Figure 11a shows the regions over which the PISFVLOF data was averaged in the spanwise direction. The FISF data points which lie in these regions are also plotted with the streamwise sections in Figure 11b. 


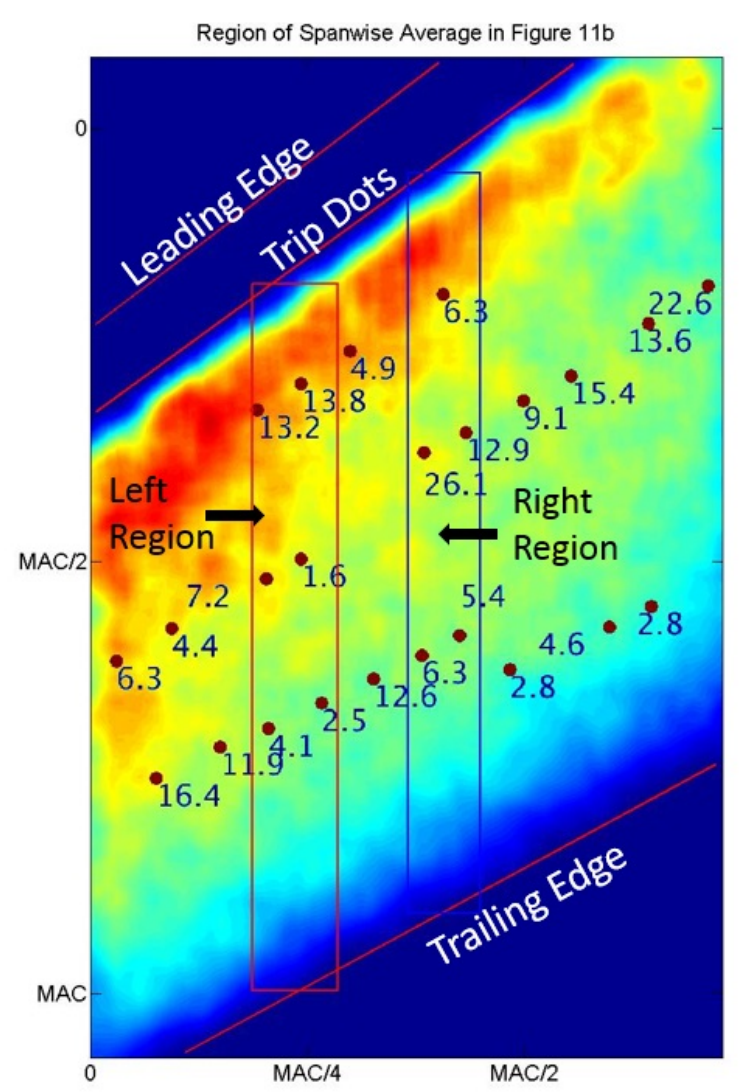

Figure 11a. Regions over which PISFV-LOF data was averaged to produce the streamwise sections shown in Figure $11 b$.

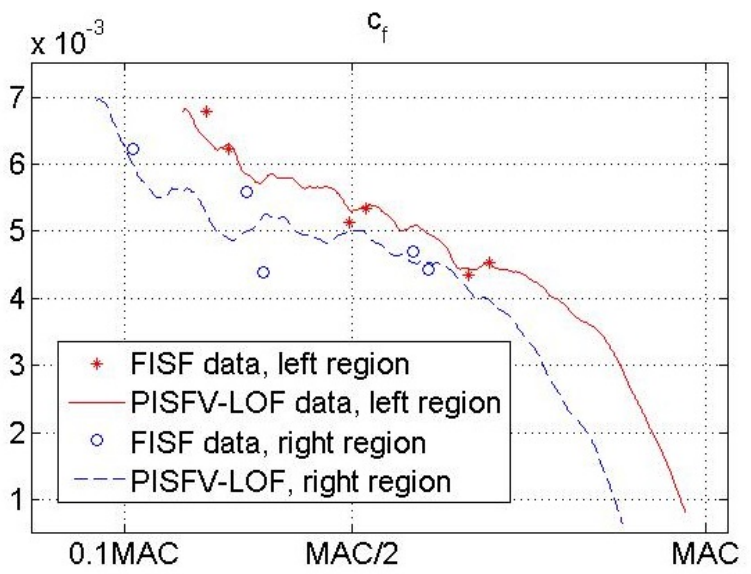

Figure $11 b$. Streamwise sections PISFV-LOF generated $C_{f}$ values were averaged in the spanwise direction over the regions shown in Figure $11 a$. The FISF generated $c_{f}$-values which fell into those regions are also plotted in this figure.

\section{Conclusion}

The Particle Image Surface Flow Visualization with Luminescent Oil-Film (PISFV-LOF) skin-friction measurement technique shows promise. The preliminary data set shows reasonable trends in skin-friction coefficient magnitudes, and the percent deviations from fringe-imaging skin-friction values are fairly low for preliminary data using a newly developed technique. Increased accuracy can likely be achieved by taking care to account for temperature fluctuations of the oil-film. The technique currently produces a relative skin-friction field. In order to resolve nominal skinfriction values from the technique an accurate oil-film thickness-luminescent intensity calibration is necessary. Further work could focus on more accurately measuring the oil-film thickness through luminescent intensities.

\section{Acknowledgements}

Special thanks to Neal Watkins, NASA Langley, for his chemistry expertise and help finding suitable dyes for this endeavor, and to the rest of the researchers at NASA Langley's Advanced Sensing and Optical Measurement Branch for their conversation and input.

\section{References}

Etebari, Ali, "Recent Innovations in Wall Shear Stress Sensor Technologies,” Recent Patents on Mechanical Engineering, 1, pp $22-28,2008$.

Kahng, Seun K., C. D. Hernandez, S. A. Gorton, "MEMS Sensor System Development at NASA Langley Research Center for Wind Tunnel Applications," $40^{\text {th }}$ AIAA Aerospace Sciences Meeting \& Exhibit, Reno NV, 2002.

Liu, T., and L. Shen, "Fluid flow and optical flow" Journal of Fluid Mechanics, Vol. 614, No. 11, pp. 253-291 (2008)

Liu, T., and Sullivan, J. P., "Luminescent Oil-Film Skin Friction Meter," AIAA Journal, Vol. 36, No. 8, 1998, pp. 1460-1465.

Liu T, Montefort J, Woodiga S, Merati P, Shen L (2008) Global luminescent oil-film skin friction meter. AIAA J 46(2):476-485

Massey, B. S., Mechanics of Fluids, London: Van Nostrand Reinhold, 1970, Chapter 6.3.

Mosharov, V., Orlov, A. and Radchenko, V. (2006) "Application of Particle Images Surface Flow. Visualization Method for Large-Scale Model," ISFV12, Proc. Of $12^{\text {th }}$ International Symposium on Flow Visualization, paper \# 361 , pp. $1-6$.

Mosharov, Vladimir, and Radchenko, Vladimir, "Visualization of Boundary Layer Transition by Viscous Oil," Visualization of Mechanical Processes, Volume 3, Issue 1, 2012.

Naughton, J. W., and Brown, J. L., "Surface Interferometric Skin-Friction Measurement Technique," AIAA Paper 962183, June 1996. 
Naughton, J. W., and M D Hind, "Multi-image oil-film interferometry skin friction measurements" Meas. Sci. Technol. Volume 24, number 12, 2013.

Naughton, J. W., and Mark Sheplak, "Modern Developments in Shear Stress Measurement," Progress In Aerospace Sciences, No 38, pp. 515 - 570, 2002

Reda, Daniel C., Michael C. Wilder, Rabindra D. Mehta, and Gregory Zilliac, "Measurement of Continuous Pressure and Shear Distributions Using Coating and Imaging Techniques," AIAA Journal 36:6, 895-899, 1998

Ross, James, C., et al, "Comprehensive Study of the Flow Around a Simplified Orion Capsule,” AIAA Paper 20132815, San Diego, CA, June 2013.

Tanner, L. H. and Blows, L. G., "A Study of the Motion of Oil Films on Surfaces in Air Flow, with Application to the Measurement of Skin Friction," Journal of Physics E: Scientific Instruments, Vol. 9, 1976, pp. 194-202.

Zilliac, G.G., "Further Developments of the Fringe-Imaging Skin Friction Technique,” NASA TM-110425, Dec 1996.

Zilliac G.G., et al., "Comparison of the Measured and Computed Skin Friction Distribution on the Common Research Model,” AIAA Paper 2011-1129, Orlando, FL, Jan 2011. 